\title{
INFORMATION, GENETICS AND ENTROPY
}

\author{
Julio ERNESTO RUBio BARRios
}

\begin{abstract}
The consolidation of the informational paradigm in molecular biology research concluded on a system to convert the epistemic object into an operational technological object and a stable epistemic product. However, the acceptance of the informational properties of genetic acids failed to clarify the meaning of the concept of information. The "information"' as a property of the genetic molecules remained as an informal notion that allows the description of the mechanism of inheritance, but it was not specified in a logic-semantic structure. The metaphorical implications associated with the idea of genes as molecules with meaning, questioned the linguistics that seemed too foreign to molecular biology. A reformulation of the concept of information in molecular biology was developed upon the theory of Claude Shannon. The node for the structural coupling between biology, physics and information theory was the identification of an analog structure between the coded messages of Shannon's theory.
\end{abstract}

Keywords: Information theory; molecular biology; Maxwell's Demon; genetic information.

\section{Introduction: information theory after Shannon}

The concept of information has become an important notion for many levels of knowledge after the research contributions of Claude Shannon $(1948 ; 1949)$ in the late forties, to optimize the processes of transmission of encoded signals. Originally, Shannon named his work mathematical theory of communication, but was replaced in practice as theory of information, due its focus on the optimization of process of coding, i.e., in the specification of the informational content of signals, rather than in the process of generation and transmission of physical signals, which is generally associated in electrical engineering with the concept of communication. The information theory is not directly about the physical signs but about the coded messages. Thus, it is possible to perform a mathematical analysis of "... the measure of information, the capacity of a communication channel for transferring information and encoding as a means to use the channels at full capacity." (Carlson \& Crilly 2009, p.698)

Shannon was interested in the design principles of systems of transmission and reception signals that minimize the probability of error in the process. Thus, he conceived a definition of information based on the probability of occurrence of a message: the information (I) was defined as the logarithm (base b) the inverse of the probability of the message (A):

$$
I=\log _{b}\left(\frac{1}{P_{A}}\right) .
$$

Principia 19(1): 121-146 (2015).

Published by NEL — Epistemology and Logic Research Group, Federal University of Santa Catarina (UFSC), Brazil. 
The basic assumptions of this definition are: (i) information depends solely on the probability of the message, not the semantic content. If a message is unlikely to happen, it contains much information, if it is very likely to happen, it contains little information. In the extreme cases, a message with a probability of one contains zero information and a message with a probability of zero contains infinite information. (ii) The definition offered is meaningless if it is possible to assign a probability to the messages, which implies the existence of a set of possible messages (sample space) where we can make the assignment of probabilities; i.e., we talk about computable a priori probabilities on the occurrence of a specified message, but a posteriori on the set of possible signals.

Shannon used mathematics to build the concept of information. The use of mathematics is common, of course, in areas that are not restricted to technological applications. Mathematics is a key element of scientific work, particularly in the natural sciences. The distinction between the purely mathematical use of mathematics and the scientific use of mathematics can be explored from the pragmatic perspective that characterizes technological production processes. Mathematics is, in a technological discipline, a calculation tool that is integrated into the production process of technical apparatus. The calculation contributes to the production of technological products. In this case, mathematics acts as the modeling language of an idealized reality. However, the distinction does not imply that there is no pragmatic use of mathematics in science, or that technology does not include elements of cognitive production. By contrast, technology modifies reality and creates significant spaces for transforming our bodies of knowledge. On the other hand, eventually, science produces knowledge as a result of technological processes by using mathematical models. Thus, the boundary between science and technology is not clear, especially in contemporary science, and the relationship is neither the reduced role of technology as a validation mechanism-experimentation nor the practical result of application of scientific knowledge.

The material production of technological objects in the laboratory according to the logic of ad-hoc dissection of nature shows the first order approximation to understand the role of technology in the construction of scientific knowledge. The molecular machinery unveiled under the constraints imposed by the devices and technological objects. Another structural coupling between science and technology occurring in the construction of the concept of genetic information is given by the analog transfer of theoretical structures generated in the information theory. In this case, we do not speak of technological restrictions embodied in the use of devices for handling experimental systems, but communication structures generated in a field of theoretical work technologically oriented, that are imported by molecular biology to make sense of an internal notion.

The information theory is a technological product as it is originally oriented to

Principia 19(1): 121-146 (2015). 
the analysis and optimization of the generative process of physical signals in communication channels as part of material handling systems of specific types of energy. Moreover, this theory is a communications product, simply because every theory is a linguistic construction that serves as a communication structure for encoding phenomena.

After its appearance in a technological context, the information theory found application in other theoretical fields. The possibility of forming particular notions in mathematical terms seems to be the main reason for the interest that other disciplines had in this theory. The language of science builds enclosed codes. Shannon's informational approach gave the opportunity to refine the concept of information in those areas that handled ideas associated with streams of coded structures. Some particular knowledge systems with such features coupled with the information theory, and restructured some of its own content based on that interaction. The use of the concept of Shannon is discussed in the analysis of social disciplines as pedagogy (Frank 1966); as the theory of psychological perception of Abraham Moles (1966); cybernetics (Wiener 1966); biology and molecular genetics (Lwoff 1962; Eigen 1992); and in certain approaches to problems of physics, such as the paradox of Maxwell's demon.

However, since 1966, the ability of the theory to produce useful analogies in science was discussed. Mandelbrot (1966, p.61) then stated that the information theory should be abandoned as a form of new interdisciplinary approach to problems: “... Turning to information theory in the strict sense, when I am asked today how to learn it to participate in interdisciplinary activities ... I respond without hesitation that it is not worth learning it."

As it usually happens in the dynamics of science, policy principles that emerge from the communicative dynamics of science itself are usually ignored. The usage of the concept of information in other fields did not stop with limiting criteria as the one offered by Mandelbrot. In molecular biology, information theory provided the structure for analog implementation of a structuralist paradigm to the interpretation of genetic information.

\section{The analog transfer of Shannon information concept in molecular biology}

Perhaps the most spectacular case of analogical transfer was the application of the concept of information of Shannon in molecular biology. The discovery of the biochemical structure of nucleic acids (DNA and RNA) in the fifties pointed out the fundamental mechanism of biological inheritance and opened a new area of research in biology. It was found that the gene sequences are formed by arbitrary chemical chains of nucleobases (four possibilities: two purines and two pyrimidines). Heredi- 
tary transmission of characters is then interpreted as a causal decoding result of the information contained in the gene sequences. It comprises four bases as the alphabet of the genetic code and genetic sequences as the development programs of biological systems. In this context, it was quite natural to apply the concept of Shannon. Genetic sequences could be seen as messages in a specified code, where the combinatorial possibilities of the alphabet could be calculated, thereby formalizing the concept of genetic information.

However, the analog transfer of the concept of information required structural conditions in the communications code of structural biology. These conditions were present in biology in relation to the issue of inheritance, which opened questions about the information content of the substance responsible for the generational transmission of characters. The question had been raised since the nineteenth century by authors such as Lamarck, Darwin himself and, of course, Mendel. These investigations continued in the work of Weismann and then with the work in molecular biology that led to the discovery of the structure of DNA and the elucidation of the mechanisms of heredity at the molecular level. The biological subsystem of inheritance was found in the structural coupling between molecular biology and information theory when the isomorphic structure between DNA and digital messages were observed, enhanced by the use of the term information to describe Shannon's theory.

Applying the definition of the information theory in the case of genetic macromolecules established the apparent conditions for the conceptual closure of an epistemic object, rarely seen in Biology. Not only the molecular structure of the substance of heredity and the sequence of biochemical reactions that turned the hereditary content into proteins was know; also the concept of information was formalized mathematically. However, the mathematization of genetic information is not easily closed. By contrast, the mathematicised definition of genetic information became the center of a controversy over the meaning of information.

\section{Semantic information and context}

In plain language, information is understood as the set of data drawn from some experience. This experience can occur with a verbal or non-verbal agent. We can get information from a newsletter and we can also get it from the observation of a tree, a landscape, or a mineral. What is the source of information? Is it found in the object of reference, in the observer, or in the process?

If we think in terms of ordinary language, we speak as if the information was found in the object and we simply withdrew it. However, the information does not result from the mere perception of the object but of a certain kind of processing of perceptual experience. When we say that an object is made up in a certain way, that

Principia 19(1): 121-146 (2015). 
is, when we describe the object, we use a verbal code to perform the conversion of specific experience into information. Of course, the code is not in the object. It is the agent who encodes an experience to produce information. Information generation requires a code; information is obtained by encoding an experience. We cannot say that reality itself carries a code, but some experiences are themselves encoded experiences (e.g. the verbal exchange between human agents). Generally, the acquisition of information requires the use of a code that allows the translation of an experience in information.

The coding involves translating the unique experience as a symbolic context of a more general level that includes a significant range of possibilities. For example, if we see a leaf and we conclude it is a green leaf, we are using a code that contains the range of colors that we can identify. Thus, the process requires a context of more general interpretation of the singular experience level coded. Bateson analyzes the structure of cybernetic explanation and claims:

The hierarchy of contexts within contexts is universal in the communicational aspect (or "emic") of phenomena and always leads the scientist to find the explanation in increasingly larger units. In physics (perhaps) it is possible that the explanation of the macroscopic should be sought in the microscopic. In cybernetics the opposite is true: there is no communication without context. (Bateson 1967, p.430)

The contextual nature, suggested by Bateson to explain cyber communication processes, is significative. Therefore, an experience with informational content makes sense within the conceptual network that the individual possesses. We cannot talk of meaning as if it were an object or thing. The meaning is not an exclusive relationship of the object with information; rather it forms from the several communication relationships the individuals have with their linguistic community and the conceptual patterns that encode their cognitive experiences.

We can conclude the semantic characterization of information by establishing the levels involved in any process of acquiring information:

1) The level of the physical object to which attribute informational content: physical phenomenon or a specific signal (an image, a sound set, or setting some kind of energy).

2) The level of signal encoding.

3) The level of contextual interpretation that gives meaning to the code.

Whatever the source of semantic information, we will find the three levels indicated: an energy demonstration, a code, and a context of code generation and interpretation. 
From the contextual meaning of the information, Bateson understood the information flow as the detection of the difference and defined information as a difference that makes a difference. The author explains:

... when there is comparison or information, for me there is a mental process ... A sense organ is an organ that compares; it is a device that responds to a difference. The body is material, but its condition is responding the difference that allows us to distinguish his performance as "mental" ... our explanations, our treaties inanimate matter, they are full of information. But that information is all ours, is part of our life processes. (Bateson, p.30)

What Bateson calls mental represents the undisposable contextual dimension of a process where information flows. Bateson is trying to build a concept of information valid for the conceptual framework of cybernetics. This discipline is concerned with self-monitoring systems that require a detection capacity of their environment and generating responses. He seems to apply in this case, the idea of the story of the difference as a property of a mechanism that is self-regulating. Furthermore, the human meaningful context required to code interpretation also points to the problem of awareness. Cybernetics is right between the organism conception of a machine and a mechanistic conception of a living being. The relevant question is then whether the nature of the information is the same in the case of humans as in the case of a mechanical device self-regulated and, more in general, any system that is capable of responding to the signal carrier differences. The answer lies in the level of the context of interpretation. The differences will arise from physical arrangement and the detector system will have a code signal processing in some form of response that executes a specific material action, but it is the nature of the code and its generative context that will be different when we talk about a feedback device, a human being, a butterfly, or the cellular machinery for decoding of the genetic code.

The informational approach in molecular biology recognizes the existence of a genetic code as a nodal point in the explanation of heredity: The regular use of terms of semantic guidance stems from such recognition. Furthermore, the information theory of Shannon provided a structure to formalize the concept of genetic and compositional information in mathematical terms. The search for meaning in the concept of information in molecular biology structurally coupled the notion of information theory and the notion of contextual information from the semantic meaning of coding. In relation to both notions, genetic information presents definite differences. Unless dismissed the particularities of human language in the dimension of communication and mental life, or established a clear sense of semantic notions in molecular biology, the allocation of significant interactions between biological macromolecules is questionable. Genetic coding does not meet the teleological condition of human semantic codes (unless we postulate a hidden teleological agent: God, nature, etc.) We

Principia 19(1): 121-146 (2015). 
cannot speak of "meanings" without distinguishing its notion in the case of the interaction between molecules. However, the semantic information and genetic information share the need of a functional approach to its interpretation. On the other hand, although the DNA has an isomorphic structure with encoded messages of Shannon, the informational description of biomolecular interactions has a contextual aspect that does not match the structural definition of the information theory of Shannon. The "contextual information" makes sense mainly in a functionalist interpretation of the system that designates the context of informational flow (semantic or biological). The "information" of Shannon is totally independent of the context (although this is necessary for code generation) and depends mainly on the compositional possibilities of the coding. The coexistence of such linguistic usages established a tension between the functionalist (contextual) and structuralist interpretation (compositional) of genetic information.

\section{Information theory and molecular biology: contextual function or structural probability?}

The concept of Shannon seemed a natural solution to the definition of genetic information, first, because the structure of macromolecules corresponded to the coded messages of the theory of information structure; secondly, because the descriptive information equation was isomorphic with the negative of the thermodynamic entropy; and third, because the mathematized definition of Shannon theory met with the Newtonian paradigm of closing the representation system operational requirements. However, the critical reactions against the scope of the Shannon equation in molecular biology soon occurred. For example, in 1962, Lwoff, who had formed part of the phage group, reacted to the ideas that began to arise in the sense of proposing the Shannon equation to measure the amount of order (defined as negative entropy and calculated as a product of the number of combinatory possibilities of the bases of the code) in an organism:

... some biologists have tried to apply the idea of negentropy ... to the living organism. The probability can be estimated so that a given sequence of nucleic acids is done by randomly mixing bases. But I think that this calculation makes no sense ... In reality, the functional aspect of the living organism must not neglect; the negentropy calculation using formulas by Shannon does not apply to living beings at all. (Lwoff 1966, p.128).

The amount of information in a DNA sequence is calculated starting from the number of possible combinations of nucleic bases. If you have $\mathrm{N}$ units in the DNA sequence, the amount of possible combinations is (4) $\mathrm{N}$ as there are 4 possibilities for each position. The amount of information is proportional to the inverse of the number 
of possibilities. Lwoff (1962) made the calculation for the bacterium Escherichia Coli, using the Boltzmann constant as constant of proportionality, and returns a value of $2 \times 10-9$ units of entropy. The relevant question is about the meaning of the number obtained in a biological context. Lwoff defends that the Shannon equation describes the functional aspect that has been traditionally used to construct explanations in biology. From this perspective, the calculation of Shannon applied to genetic sequences says absolutely nothing about the biological operation.

When calculating the value of entropy in a channel of communication in a flow of scrambled signals, calculation of the information in a message is a measure of its improbability (or probability, if preferred). The process of communication of signals takes place in the time and the amount of information of a given message makes sense in the assembly of transmitted signals. The amount of information the signal has tells us the frequency of occurrence of the message. However, there is not an equivalent phenomenon in the biomolecular case. The amount of information in a DNA sequence does not measure the frequency of occurrence of the message contained in a particular sequence, as if this was the case, the probability of occurrence of the message is practically equal to one; given the internal processes of an organism, the DNA remains almost immutable. Therefore, calculated in terms of Shannon's information, it is practically equal to zero. It would be necessary to specify the assembly of possible messages to assign probabilities of occurrence and thus obtain a spreadsheet with some sense. The amount of information obtained on the basis of nucleic combinations makes no sense in the original equation of Shannon because there is an ensemble of possible DNA sequences, where the sequence information tells us which is the probability of occurrence of the assembly.

The functional specificity of the macromolecular order cannot be ignored on the clarification of the meaning of the concept of genetic information. Lwoff dramatically illustrates this point in the case of mutations:

As a result of an accident, sometimes the nucleic base of the genetic material is replaced by another nucleic base. This alteration may cause the death of the organism. If, for example, a given molecule of guanine in a gene is replaced by a molecule of adenine, the information, the negentropy of the system, is the same. For the physicist, nothing has changed even if the mutation is lethal: the content of negentropy remains the same. (Lwoff 1962, p.113).

As we see, the argument of Lwoff shows that the meaning of genetic information presents a dimension that is not present in the mere structural order of the physical system. Does genetic information in a biologically inoperative DNA sequence exist?

When we refer to the information contained in the DNA we assume the possibility of generation of an organism from a minimal organizational structure that contains a biologically active DNA sequence. An arbitrary sequence of DNA or RNA that is not 
involved in the generation of a functional biological system may not be designated as genetic information. However, the sequence would still have a number of associated information, in the sense of Shannon, although it lacked direction in biological terms.

We conclude that the application of the equation of Shannon to the calculation of the informational content of genetic macromolecules does not produce any relevant knowledge of the place of information in the biological order, specific and functional. The use of the equation of Shannon to try to quantify the genetic information is wrongly based on a confusion of the type of working contexts in both cases. It is not possible to move the statistical context, which gives meaning to information in the theory of Shannon, to the functional context of molecular biology.

\section{The analog transfer of the concept of information in Shannon to physics}

Apparently, the unveiling of the molecular mechanism of inheritance had shown the absence of new laws of physics that had been suggested by Bohr and sought by the phage group. Thereby, the possibility of reducing biology to physics was excluded in the specific field of molecular genetics, where the possibilities of an inter-theoretical reductionism have a natural test territory, because they occur in conditions of maximum structural specification and minimum level of functional order. The mechanisms of transfer of genetic information seemed to demonstrate the irreducible functional nature of biological operations, even at the molecular level. The terminology proposed by Zuckerland and company highlighted the functional order of operation of genetic macromolecules: If the DNA contained coded information that regulated the organismic order, other molecules would have the function of decoding the message to the rest of the body.

Nevertheless, the application of the mathematical definition of the theory of Shannon of information to the genetic case revived the reductionist tension between physics and biology. Science pursues closed models of linguistic reconstruction of the objects of study. In this sense, classical physics provided a model of science which was based on the construction of closed systems of variables, which are known as the Newtonian paradigm (Rosen 1985; Martinez 1990). The implementation of this model of representational construction can be seen in the case of the concept of genetic information such as the application of compositional and structural model of the information theory to the molecular case.

However, the application of compositional paradigm was not the only way of communication link between physics and molecular biology. Based on the definitions provided by Shannon, a new defense aroused on the possibility of eventually reducing biological theories to physical-chemical theories. The reduction of biology 
to physics found a theoretical exploration area in the existence of other structural isomorphism, this time not between digital message and genetic sequence, but between the mathematical form of the equation to calculate the amount of information and the equation to calculate the entropy of a system. Entropy is associated with the amount of existing disorder in a thermodynamic system; on the other hand, the genetic information could be seen as the origin of the biological order. Thus, genetic information raises as a measure of the thermodynamic conversion of physically disordered systems to biologically organized systems.

The relationship between entropy and information did not initially appear in relation to the specific problem of the genetic information. In 1929, Szilard proposed a relationship between the production of entropy and the intervention of intelligent beings in the functioning of devices that materialize the paradoxical behavior of systems where the hypothetical "Maxwell's demon" reduced entropy. Then, the problem was addressed by several theorists (for example Brillouin 2003; Schrödinger 1944) that explicitly introduced the concept of information of Shannon in relation to the problem of Maxwell's demon. It is not until after the speculation made about Maxwell's demon that the concept of information of Shannon applies to biological contexts and, later, to the molecular genetic sequences.

The classic treatment of Szilard is not directly used, however, from the concept of information, as its work is interpreted a posteriori. Szilard wants to show that the intervention of intelligent beings in a decreased entropy device by intervention of Maxwell's demon necessarily produces an amount of entropy of a magnitude at least equal to the decrease of the entropy of the system. To structure his argument, Szilard talks about processes of measurement (measurement), memory (memory) or coupling parameters (coupling) and only mentions the notion of information in the introduction of his article:

A perpetual motion machine therefore is possible if - according to the general method of physics - we view the experimenting man as a sort of deus ex machina, one who is continuously informed of the existing state of nature and who is able to start or interrupt the macroscopic course of nature at any moment without the expenditure of work. (Szilard 2003, p.111).

In his demonstration, Szilard formalizes the notion of measurement as a link between variables that represent, on the one hand, the state of the system and, on the other hand, the state of the measuring device. The notion of Shannon, who will be shown until 1948, does not have a direct antecedent in the work of Szilard but will later come to be part of the discussion and even will be attributed to the own Szilard. For example, Brillouin (2003, p.134) writes about the paradox of Maxwell's demon: "The paradox was considered by generations of physicists, without much progress in the discussion, until Szilard pointed out that the demon actually transforms "information" into negative entropy."

Principia 19(1): 121-146 (2015). 
Despite the subsequent interpretation, Szilard never makes such a specific statement in his work. Conversely, Szilard rigorously defines the conditions of his demonstration, rejecting the formalization of intelligent or biological systems and replacing the participation of these by physically moldable devices. Szilard works a thermodynamic problem in thermodynamic terms. The concept of entropy that Szilard talks about is the one belonging to physics and does not intend an equivalence between information (or intelligence or other condition of consciousness) and negative entropy. Its explicit reference to biological systems, however, clearly opens the application of this demonstration to such systems and points out the conceptual ground for the coupling that would occur later between the information theory and molecular biology.

The link between physics and information theory is not exclusively generated from the informal suggestion of Szilard about the conversion of information in negative entropy. Drawing on information theory, Shannon drives analog identification between information and negative entropy, building its definition of entropy in communications from the isomorphism between thermodynamic entropy equation and the descriptive equations of the systems of signals. The equation for entropy in thermodynamics and in information theory is

$$
H=-k \sum P i \log P i
$$

Where $k$ is a constant, and $P i$ represents the probability of occurrence of a microstate (in thermodynamics) or the probability of occurrence of a message (in information theory).

Such isomorphism had already appeared since 1928 in the equations that Hartley had developed for the transmission of telegraphic information. Two decades later, Shannon resumed and developed the notion of Hartley to turn it into an elegant theory about the transmission of coded messages. However, the isomorphism between equations is not sufficient explanation for a same term applying to two equations that represent different phenomena. The choice of the term "entropy" by Shannon responded also to specifically communication interests. By recommendation of von Bauer, Shannon chose the term "entropy" (Wicken 1987), in order to exploit the semantic load that the term already possessed in physics.

The communication strategy of von Bauer had an undeniable and immediate success. Wiener (1948) appears to be the first to propose the identification of information and negative entropy. The context of work is cybernetics, and the notion of information is that which applies to feedbacked systems. A cybernetic system behaves teleonomically according to their ability to capture a signal that indicates the suitability of the system with its expected output. The feedbacked signal is compared with the expected level and the behavior of the system is modified in relation to the result of the comparison. The system then holds its operational order using energy to 
constantly check its status. The information could be associated in cybernetics with physical operational flows in specific systems and a mathematical definition would have the ability to close the system of concepts of the nascent cybernetics in such a way that it establishes validatory ties to physics, as well as providing a concept which also expresses the order of biological and psychological systems that cybernetics was about to model.

After the emergence of the theory of Shannon, the association between information and negative entropy became a reference point for the discussion on the nature of the order from the physical layer to the social level. In the case of the relation between physics and biology, information theory functioned as a bridge for the structural link between these two disciplines that were found at a conflict of the reductionist type. The identification of information and negative entropy has been a point of conjunction that has kept this fundamental conflict.

\section{The interdisciplinary link between information theory, physics and biology: the concept of entropy}

The identification between information and negative entropy (thermodynamics) faces a tension itself where biology does not necessarily appear. In 1951, Brillouin had noted the problem of the identification of information (encrypted) and negative entropy (physical) in unique objects that match in the absolute amount of negative entropy. Speaking about the approaches made in Wiener cybernetics, Brillouin states:

Take an issue of the New York Times, the book on Cybernetics, and unequal weight of scrap paper. Do they have the same entropy? According to the usual physical definition, the answer is "yes." But for an intelligent reader, the amount of information contained in these three bunches of paper is very different. If "information means negative entropy," as suggested by Wiener, how are we going to measure the new contribution to entropy? (Brillouin 2003, p.123).

The identification of information and negative entropy is the target of the claim by Brillouin: the specificity of the value of information (encoded) can be very different in individual cases, however, they may have the same physical entropy. ${ }^{1}$ The mere linguistic identification of entropy with disordered and ordered information is at the heart of this tension. The tension has remained as the epistemic object of a research system that has produced original theoretical constructs and generated another structural coupling between physics and biology.

Layzer (1990) proposed an important modification to the original identification of information and negative entropy. Layzer notes that it is not possible to formally compare different manifestations of order (physical, biological, linguistic, etc.), but 
that it is possible to identify a general manifestation of disorder. The order in each type and level of organization is specific, but the disorder is the same. On this premise, Layzer proposes that information, as a notion of order, cannot be understood as mere negative entropy, but can be formulated in terms of disorder of a system. The information is not directly dependent on the entropy of the system, but on the difference between the maximum entropy (maximum disorder) and the observed entropy (disorder de facto):

Randomness is a numerical measure of disorder. In the same way, information, defined by the formula Information = maximum randomness - actual randomness is a numerical measure of order. Information is thus potential randomness... Processes that increase randomness destroys information; processes that decrease randomness generates information. (Layzer 1990, p.27-8).

Therefore, the condition of randomness is defined as the logarithm of the number of micro-states which correspond to a given microstate. This is precisely the physical definition of entropy, developed by Boltzmann in 1873, where the microstates of the system are established based on a collection of dynamic variables.

Layzer applies his definition to genetic macromolecules. According to Layzer, the direct application of Shannon's formula to this case mixes up macrostates with microstates, and only a few combinations of bases are functionally adequate and therefore contain information. The other combinations are informationally empty. The author identifies the macrostates of the sequence with the functionally adapted combinations of nucleobases and microstates, with possible combinations of bases. Thus, Layzer attempts to capture the biological functionality in its equation for calculating information and tries to overcome the constant critique to the application of Shannon's equation in a biological context. Layzer tries to clarify the application of Boltzmann's equation of physical entropy in molecular genetics. But the phenomenical relationship between information and entropy is not elaborated by Layzer. The author seems to suggest that the relationship is simply given by the mathematical structure and not by their meaningful content, even though in relation to the implementation of the Boltzmann formula to genetics, Layzer (1990, p.31) says: “... But Boltzmann's definition of randomness, like every mathematical definition, is a pure form, devoid of content. Let's see if we can't find an appropriate biological content for it."

However, the formulation of Layzer fails to clearly establish the relationship between the proposed equations and the biological interpretation of the equations. The phenomenic distinction between thermodynamic, biological and informational order is entered by the author, but the confusion of levels of organization remains on the equivalence of randomness of different levels. The direction toward a conceptual unification closure of system overrides the distinction of phenomenic levels. 
Anyway, Layzer makes a central contribution to the research system of information: the distinction between macrostates and microstates. This distinction operated as a variation of the reproduction of the research system that allowed new developments around the problem of information.

Using the ideas of Layzer and others (especially Collier), Wiley and Brooks attempted to develop a formal apparatus to establish the relationships between information and entropy. Actually, the intention of these authors goes far beyond the relationship between information and entropy. Wiley and Brooks tried to build a general theory of entropy as the top unified principle at all levels of organization in biology. For this purpose, they developed a hierarchical information theory that tries to overcome the shortcomings of the somewhat crude application that was at the origin of the identification between entropy and information. Wiley and Brooks (1986, p.32-3) take the basic idea of Layzer on the distinction between macrostates and microstates and develop a formalism that encompasses different levels of nested organization and thus seek to solve the physical relationships between biological information and entropy: “... biological information (in all of its hierarchical manifestations) is subject to the constraints of the second law of thermodynamics in the same way that energy flows are subject to the law in its more traditional thermodynamic manifestations."

The issue of genetic information appears again in the work of Brooks and Wiley. To the authors, the definition of Shannon seems insufficient as a complete description of biological information and thus they construct a typology that distinguishes genetic information as "Instructional Information" (following the terminology of Collier). The instructional information is a physical condition of DNA that is postulated as an intrinsic expression of organisms; that is, it is a self-referential condition of the body that is not structured by the environment, but only by the system itself. Furthermore, the instructional information of the DNA is a physical condition of the system that is not dependent on the observer. Unlike the semantic interpretation of the information, the instructional information, according to the authors, does not require to be defined by any observer. This type of information is an accessible material condition in the internal processes of organisms. The explanation is most relevant to the overall purpose of the theory of Brooks and Wiley. To seek specific relationships between biological and thermodynamic entropy, the information must be contemplated in physicalist terms. The physicalization of biological information appears to the authors as the means for the closure of the historic system of relations between information and entropy. The system of relations that Wiley and Brooks proposed includes the concept of information of Shannon, seeking the closure of relations between three disciplines (information theory, biology and physics). Wiley and Brooks (1986, p.91) then defined the operation of biological information as a communication system: "Because biological arrays are also communication systems, biological 
array information is also a subset of message information (from communications theory)..."

The concept of information used by Brooks and Wiley arises from the association of information theory, biology and physics. The novelty found in the theory of Wiley and Brooks is the level of development of his theoretical work and epistemological self-consciousness. The authors start from the information theory of Shannon, but complement the definitions using the distinction between macrostates and microstates of Layzer, which does not appear in the work of Shannon and Brillion. Also, they try to capture the complexity of biological systems considering the hierarchical organization of the participating levels of organization, and therefore they attempt to take the relationship between information and entropy as far as it will go. The hierarchical theory of information developed by Wiley and Brooks proposes a series of distinctions between classes of information that serve the purpose of generating formal analogies to establish the bridge between physics and biology principles:

\begin{abstract}
As a formal analogy with statistical thermodynamics, HIT [hierarchical information theory] implies a number of terminological equivalences... The total information capacity of the system is equivalent to the total entropy (Hmax) of the system. The information content of the constraints is equivalent to the entropy of the constraints (Hmax-Hobs), or to the macroscopic information..., or to the macroscopic portion of the total entropy... The information content of the system is equivalent to the entropy of the system (Hobs) or to the microscopic information... or to the microscopic portion of the total entropy... We must also show that these equivalences are useful and meaningful. (Wiley and Brooks 1986, p.72-3)
\end{abstract}

The theoretical project of Wiley and Brooks can be classified among the great physicalist unifying projects in the same way that Oppenheim and Putnam (1958) postulated in their classic article "Unity of Science as a Working Hypothesis" an epistemological unifying project where the scientific theories of any specific level of organization are supported by theories in the lower level of organization, so that physics can sustain any scientific theory. Wiley and Brooks would be contributing to this project if they actually succeeded in the unification of physics and biology. The identification between information and entropy would be the main bridge allowing reduction. In addition, the epistemic object of the research system on the relationship between genetic information and entropy could reach the reductionist closure sought by Brillouin, Wiener and others. Despite these noble purposes, the system is far from closure in the theory of Brooks and Wiley for the reasons explained below.

Wicken (1987, p.177) clearly presented the problem of interdisciplinary communication in the case of the relationship of information-entropy. The concept of entropy, the author tells us, has gone through several theoretical fields that have different significant uses of the term. The notion of entropy in classical thermodynamics indicates 
the restricted direction of the dynamics of a macroscopic system. The entropy (S) was defined by Clasius as the ratio of heat $(\mathrm{Q})$ to temperature (T), as follows:

$$
d S=d Q / T
$$

The second law of thermodynamics postulates that this ratio always increases in any irreversible process. This is the original meaning of the term "entropy" a generalization of the concept, stated by Wicken, should share this fundamental characteristic.

Boltzmann introduces in thermodynamics the distinction between macrostates and microstates as a way to build a causal explanation that departed from the dynamics of microscopic components (molecular) of a system. Thus, Boltzmann equation can be used to mathematically derive the macroscopic formula by Clasius:

$$
H=k \log W
$$

where $k$ is the Boltzmann constant and $\log W$ is the natural logarithm of the number of equiprobable microstates corresponding to the macrostate of the observed system. After Boltzmann, Gibbs extends the result to non-equiprobable microstates:

$$
H=-\log k \sum P i
$$

where $P i$ is the probability of occurrence of each microstate $i$. This mathematical form of physical entropy is what sets the formal analogy with the equation of entropy of the information theory, as shown in textbooks on the theory of communication:

$$
H=\sum P i \log 2\left(\frac{1}{P i}\right)
$$

In this formula, $P i$ is the probability of occurrence of each message in the system, the entropy $H$ is the average amount of information in the signal system. The equation is the same, since $k$ is a proportionality constant that adjusts the units system, the base of the logarithm is arbitrary and $\log 2\left(\frac{1}{P i}\right)=-\log 2 P i$.

The mathematical analogy between this equation and the formula by Gibbs provides structural support to the relationship between information and entropy. However, the mathematical equivalence would not necessarily ensure the conceptual equivalence, as noted by Wicken. The conceptual content of the equations, in information theory and statistical mechanics, is fundamentally different, the author argues. Wicken indicates that the thermodynamic states are kinetic states of particles in a system whose uncertainty lies in the statistical fluctuation of the particles through the space of phase. In contrast, the probabilities of the information theory indicate the uncertainty of the ensemble of possible messages, but once a message 
has been sent, the uncertainty disappears as a condition of the state of the message. On the contrary, the microstate of the thermodynamic particles remains fluctuating in the space of phase. Wicken concludes that the distinction between microstate and macrostate applied in statistical mechanics is meaningless to the theory of information.

The same distinction between macrostate and microstate shows the conceptual difference between the thermodynamic entropy and entropy in the information theory. Wicken states:

What allows us to assign thermodynamic system entropy is that any measurable macrostate in which it resides can be expressed in a variety of alternative microstates. Since these are all accessible by the system, there is an essential uncertainty in knowing its microstate at any instant... A message, in contrast, cannot possess entropy. It is what has been said, a fait accompli. (Wicken 1987, p.181)

Wicken indicates the fundamental role of uncertainty of the thermodynamic microstates to show that without such microstates, the informational entropy is conceptually outside thermodynamic entropy. The messages of the information theory are defined structures without alternative microstates. There is therefore no fundamental relation between microstate and macrostate that gives meaning to the concept of thermodynamic entropy, and it is not possible to use the formal analogy to establish a phenomic correspondence. The specifiable structures lack of alternative microstates and therefore its entropy pair is zero. Such is the case of a specified message in the information theory or in a genetic sequence: they do not have thermodynamic entropy.

Furthermore, the entropy of an informational system, according to Shannon's formula, is a statistical property of an assembly which measures the average expectation of occurrence of the messages in the assembly. Such a notion is not applicable to the thermodynamic context because it does not capture the distinction of macrostatemicrostate that Wicken already designated as the fundamental condition of entropy in statistical mechanics. It must add that it is not the case of gene sequences in biology. The genetic macromolecules are stable structures. The statistical ensemble of genetic messages does not exist. The DNA is a "message" or a set of messages that functionally operate in processes defined by the molecular structure and not in statistical processes.

Therefore, the double translation across thermodynamics entropy, informational entropy and genetic entropy is set on invalid intertheoretical maps. The identification of information and negative entropy mixes different phenomenic levels. The amount of information measured by Shannon's formula is applied to sequences of encoded statistical assemblies. The information depends on the number of units used for encoding and the number of possible messages, and their probability of occurrence. 
The entropy of the information theory does not quantify a physical property of the signals, but a mathematical property of the group of messages; i.e., the negative entropy of Shannon is the property of a mathematical-logical array of signs whose physical structure is irrelevant. This phenomenic confusion adds a second problematic dimension to the identification of negative entropy and information. Shannon's entropy would be the same in the assembly of physically materialized signals by an alphabet of electromagnetic waves of different frequencies, smoke signals of different duration or different size vessels. As long as the alphabet is used in a statistical process of emission of the encoded signals, the Shannon entropy measures a property of the system of signals and has no direct relation with the physical entropy of the system of physical signals used.

The use of the mathematicized definition of information to genetic macromolecules accuses both problems. The "communication" between biological macromolecules is not a statistical emission process with different probabilities assigned. The probabilities of every interaction between molecules are practically one since each context functionally determines the ongoing interaction. On the other hand, the informational entropy of genetic sequences, calculated from the structure units assigned as signals of the code, would be the same although the basic alphabet was not formed by nucleic acids but by flags, while the length of the string of base signals is the same and the corresponding probabilities for each symbol are also the same. Obviously, the physical entropy of each arrangement has nothing to do with the structure of the signs. The thermodynamic entropy is a property that makes sense only at the physical level. The entropy (or average information) of a statistical ensemble of signals only makes sense in the level of organization of mathematical representation. Genetic information is a property of a system that exists at the intermediate level of organization between the two previous ones and does not belong in any of them. Why then identify the persistence between information and entropy (negative)? We can suggest that the use of Shannon's equations allows mathematical formalization of concepts that their functional nature opposes the structural reduction. The mathematical definition allows the closure of the conceptual system under conditions of scientific nature imposed by the Newtonian paradigm of explanation: identifying state variables and establishing equations of the system. In addition, the mathematical formalization of genetic information seems to overarch the biological principles in physical principles, apparently resolving the reductionist tension between physics and biology that has accompanied the history of molecular biology.

The concept of entropy is the means of exchange of communication used to pursue the closure of a system of scientific communication. This is evidenced by the reasons which led Shannon to use the term "entropy" in his theory. Later, Wicken explains: 
To be fair: "entropy" was not a term that Shannon himself had decided on simply by virtue of the formal similarity of his equation to Boltzmann's... he did so at the urging of von Newmann for very practical reasons. Entropy was an established concept that his theory could draw on for salability to the scientific community. (Wicken 1987, p.183)

The reductionist principles of scientific work have operated as mechanisms for the operational closure by establishing very specific expectations about the structure of knowledge. One of these structures, especially accompanying the natural sciences, ${ }^{2}$ is the Newtonian paradigm of explanation. The construction of the concept of genetic information incorporated this structure of expectations when transferring the mathematicized definition of Shannon from the information theory to molecular genetics. Another structure of expectations in science comes from the reductionist trends that propose unifying projects of theories belonging to different levels of organization. This is also the case in the history of genetic information. The analog relations that are established between informational entropy and thermodynamic entropy have the purpose to find the mechanism of unification between physics and biology. The sociological force of these expectations can be so strong that it resists, as happened in the case of the concept of genetic information, the individual conditioning of epistemic structures of the theoretical fields involved. The analogies that have led to the informational and negentropic conceptions of genetic information could follow its own dynamics after being transferred from physics and information theory, but the structure of reductionist expectations have remained in the system of information research.

\section{Recent research in genetic information: the functional-structural tension and the semantic metaphor}

This section will address some recent ideas about genetic information, in order to show that research on the biological nature of genetic information has maintained in recent decades ${ }^{3}$ the tension between the structural approach and the functional approach.

Shannon's information theory found a space for application in the study of the origin of life and the modeling of the properties of living systems. ${ }^{4}$ Recently, some authors have concentrated their ideas about the origin of life around the concept of information because it allows, apparently, conceptual relationships between the level of physical-chemical organization and the biological level, through the concept of negative entropy, and it offers a formal platform for modeling primitive entities based on units of encoding strings. Manfred Eigen (1992; Schuster 1979; et al. 1981) postulated a theory about the origin of life which is known as the hipercycle theory.

Principia 19(1): 121-146 (2015). 
In this theory, the concept of information is placed as the key to understanding the emergence of life on Earth. Eigen equalizes the origin of life and the origin of genetic information and characterizes the process of molecular evolution in informational terms. From this starting point, the author tries to construct an explanation of the origin of life based on physical and chemical principles.

Eigen structures the problem of the origin of life on the basis of the concept of information: the problem of the origin of life is equivalent to finding the origin of genetic information. In addition, information of the biological entities arises, according to Eigen, under the extension of the principle of natural selection to protobiological molecular systems. The problem the author encounters is the unveiling of the organizing principle that leads to the emergence of life. The theory is called hipercycle theory in reference to the mechanism of primordial interaction between genetic sequences and peptide (enzymes), given in cycles of replication within cycles of higher order (hipercycles), where each sequence is involved in the reaction of replication of other sequence components. This setting proposes the action of selective forces on populations of primitive sequences still formed by physicochemical states lacking of biological information. Evolution then appears at the molecular level and leads to the selection and optimization of the primordial genetic chains. The concept of information is the conceptual axis of this development. In fact, the origin of life is made equivalent to the emergence of genetic information: "All the varieties of life have a common origin. This origin is the information that, in all living beings, is organized according to the same principle." (Eigen 1992, p.16-7)

Eigen defines the combinatorial possibilities of sequences of molecular bases as informational states of the system. Each informational state of the system corresponds to a sequence of bases with informational content that can be calculated using the equations of the theory of information. In addition, this conception of information allows the construction of a state-space system where possible states are represented, and it also allows the use of differential equations that model the entities that are used as potential precursors of organic life. Specifically, the construction of statistical populations of sequences called quasispecies, and statistical populations of quasispecies called hypercycles.

However, despite reductionist intentions of the hypercycle theory, the author acknowledges the limit imposed to the notion of absolute information in theory of Shannon, because it has not been possible to characterize the informational content of the genetic sequences so to satisfactorily suggest the molecular interaction between genetic sequences and functional molecules, without resorting to some principle that is not contained in the polynucleotides molecular structure.

The attractive aspect of the concept of information of Shannon lies in its structural formula of mathematized states. This allowed for the adaptation of the theory of the hypercycle to the Newtonian paradigm of explanation that entails the possibility of 
decomposition of a system.

Charles Bennett (1985) explains the tendency to privilege structural explanations in terms of states over functionalist ones. Consequently, Bennett coined a definition of biological organization based on the analogy between a biological system and the calculation capacity of a machine (for example, Turing machine) to model the generative process of any system. ${ }^{5}$ Bennet $(1985$, p.217) works to define an organized system as one that is likely to be modeled in a universal computer machine, given the initial states of the system, but disqualifies its own definition for not being posed in structural terms of the state: “... computational universality is an unsuitable complexity measure for our purposes because it is a functional property of systems rather than a structural property of states."

The Newtonian paradigm forcefully explains the possibility of obtaining the closing conditions of the models made by the definition of closed groups of state variables that allow stable handling of the model (Earman, 1985). In the end, Eigen's theory of hypercycle fails to resolve the tension between the functional or structural nature of genetic information. The author presents the equation for Shannon as a chance to formally address the problem of the origin of genetic information, and he even traces the linkages of the problem with the identification of entropy and negative information, but he fails to give any concrete application to the calculation of the amount of Shannon information. Even Eigen states the limitation of the Shannon equation to describe the biological behavior at the molecular level. In terms of statistical rules of behavior of genetic sequences — as messages written in a code- Eigen (1992, p.12) asserts: "There are also grammatical and syntactical rules. The spectrum of rules and conditions covers even the meaning of the sentence, that is, the semantic information, which is dependent upon specific premises that cannot be described by general statistical frequency laws."

On the one hand, Eigen claims the possibility of reducing the origin of life to physical and chemical laws; on the other hand, he declares the limitation of the statistical description (based on the theory of information) to describe the nature of genetic information. The strain that appears in the theory of Shannon is not an accident of the theory itself; it is the result of the original scientific-communicational conditions. Eigen theory inherits the tension, which he fails to resolve, between the functional approach and the structural approach to the interpretation of the biological behavior of genetic macromolecules. The notions of Eigen show linguistic metaphor permanence as a description of the informational-biologic order. Again it is a semantic dimension of biomolecular behavior. The linguistic metaphor expresses the contextual meaning of the term "information" and the seemingly irreducible functional order of the biological operation.

Another recent attempt to formalize the concept of genetic information has been brought up by Bernd-Oiaf Küppers (1990) in the book Information and the Origin 
of Life. The ideas presented by Küppers show us the continuity of tension between structural and functional explanation around the concept of information in molecular biology. Küppers proposes the existence of three aspects of information: syntactic, semantic and pragmatic. The syntactic aspect includes the structure and quantifiable information possibilities; the semantic aspect points to the significant contents of information allowing the functional integration of the various elements of a biological system; and the pragmatic aspect considers observable mechanisms of operation of the informational structures.

The formulation of Shannon persists in Küppers approach, although its applicability is explicitly restricted to a syntactic description of biological information; that is, to its structural aspect. The genome of an organism has a characteristic length that allows the use of the equation of Shannon on the basis of the number of alternatives that can be formed with the same length. The persistence of the equations of Shannon can be explained for the same reason of background that we have already mentioned: the weight of the Newtonian paradigm of science as unquestionable communicational structure. Küppers (1990, p.33) declares the usefulness of the definition of Shannon on the possibility of the mathematization of the problem: "In order to make an information-processing operation accessible to mathematical analysis, we need a quantitative measure of the amount of information that is contained in the sequence of symbols."

However - Küppers clarifies-, the extent of the information provided by the Shannon equation is not very useful in the treatment of biological order. Using the sequence of genetic basis of Escherichia Coli bacteria as an example, the author states:

... Yet only a few of these carry biologically "meaningful" information, that is, information that can guarantee the sustenance of the functional order of the bacterial cell. We now begin to see that the structural information in a nucleotide chain that is given by the Shannon information measure is hardly able to make useful statements about biological complexity and order. (Küppers 1990, p.48).

This argument is not new. In fact, it is an old argument that appeared with the first attempts to apply the theory of molecular biology information, explicated by Lwoff since 1962. The quote of Küppers enables us to see that the functional-structural tension of the concept of genetic information has remained fundamentally in the same terms. Similarly, it allows us to explain the return of semantic metaphors as a means of pointing the irreducible functional order of biological structures. Küppers exposes it in the following way:

Everything about biological structures that works "according to a plan," that is, everything that is controlled by information, has a "meaning" and a "significance" in the context of the functional order that we find in living systems.

Principia 19(1): 121-146 (2015). 
This means that biological information is associated with defined semantics. When in the rest of this chapter we refer to "biological" information, it will be precisely this semantic aspect of information what is meant. (Küppers 1990, p.31).

The linguistic categories employed by Küppers appear in the conceptualization of genetic information, supported by epistemological awareness. The sense of the semantic order is, as we have said, precisely to rescue the functional order of information.

In addition to the cases of Eigen and Küppers, we can mention at least another key author in contemporary theoretical biology which also makes use of linguistic categories to deal with the problem of biological information: Stuart Kauffman (1992; 1993). Kauffmann elaborates a theory of large proportions that tries to approach to a series of problems of biology in novel ways: the origin of life, the ontogenetic development of organisms and, in general, the processes of generation and continuity of the self-organization of living beings. The concept of information is approached from a semantic perspective. Kauffman gives us another approach to the problem of the origin of life (the model of autocatalytic groups of polymers) that is based on informational considerations inspired by the hypercycle theory and the concept of information of Shannon. In his theory, Kauffman prepared a concept of rules of interaction between molecular compounds called random grammars. Again, the linguistic metaphor appears as a means of representing the functional order of biomolecular behavior. Kauffman proposes systems of polynucleotides and polypeptides to interact according to a system of open rules that transform into the evolution of the system as a whole, avoiding the emphasis on informational master molecule that seems to be the most serious obstacle to the robustness of the theory of the hypercycle (Wicken 1985).

\section{Conclusion}

Although the Newtonian paradigm continues to operate as a model of science that proposes the construction of mathematical phase spaces as a translation of the object of research, the application of the equation of Shannon (or its products: the equations of Layzer or Wiley and Brooks) is constantly confronted by the specifically functional order of biological information. The limitation of mathematical-structural approaches to describe the biological behavior at the molecular level and the failure of reductionist projects to subordinate the biological explanation to the physicalist explanation have given room for linguistic analogies in the treatment of the information problem. In human language, the semantic content of symbolic exchange is functional because the participating subjects in a communication process are struc-

Principia 19(1): 121-146 (2015). 
turally coupled through the meanings shared in such a way that an emergent system - the same communication - is held on the functional coupling of the parties. Language is the structure that maintains the continuity of the communication process. In the same way, the (structural) syntactic aspect of biological information is functionally subordinated to its semantic aspect.

The research system that has held the question of the nature of genetic information as epistemic object did not resolve the tension between the functional and structural approach to their object of study. The theory of the information has provided the best elements of communicational exchange entrenched in the structural approach. However, the equations of Shannon have failed to be interpreted in a specific biological sense. On the other hand, the semantic metaphor has provided better entrenched communication exchange elements of the functionalist approach. However, neither of the concepts of linguistic origin have reached the point of conceptual resonance that will allow its entrenchment in an exclusively biological sense.

\section{References}

Bateson, G. 1985. La explicación cibernética. In: Pasos hacia una ecología de la mente. México: Planeta.

Bennet, C. 1988. Dissipation, information, computational complexity and the definition of organization. In: D. Pines (ed.) Emerging Synthesis in Science. Santa Fe: Westview Press.

Brillouin, L. 2003. Maxwell's demon cannot operate: information and entropy. In: H. Leff; A. Rex (eds.) Maxwell's Demon: Entropy, Classical and Quantum Information, Computing. London: Institute of Physics Publishing.

Carlson, A. B.; Crilly, P. 2009. Communications Systems. New York: McGraw-Hill

Earman, J. 1985. A Primer on Determinism. Dordrecht: Reidel Publishing Company.

Eigen, M. 1992. Steps towards Life: a perspective on Evolution. Oxford: Oxford University Press.

Eugen, M; Schuster, P. 1979. The Hypercycle: A principle of natural self-organization. Heidelberg: Springer-Verlag.

Frank, H. 1966. Información y pedagogía. In: M. Gueroult (ed.) El concepto de información en la ciencia contemporánea. Coloquios de Royaumount. Barcelona: Siglo XXI.

Kauffman, S. A. 1993. The origins of order. Self-organization and selection in evolution. Oxford: Oxford University Press.

Küppers, B. O. 1990. Information and the Origin of Life. Cambridge: MIT Press.

Layzer, D. 1990. Cosmogenesis. The growth of order in the universe. Oxford: Oxford University Press.

Lwoff, A. 1962. Biological order. Cambridge: MIT Press.

Mandelbrot, B. 1966. ¿Es útil "todavía" la teoría de la información? In: M. Gueroult (ed.) El concepto de información en la ciencia contemporánea. Coloquios de Royaumount. Barcelona: Siglo XXI.

Martínez, S. 1990. Más allá de la presuposición Newtoniana: propiedades genuinamente disposicionales en la mecánica cuántica. Crítica 22(66): 25-37.

Principia 19(1): 121-146 (2015). 
Moles, A. 1966. Teoría informacional de la percepción. In: M. Gueroult (ed.) El concepto de información en la ciencia contemporánea. Coloquios de Royaumount. Barcelona: Siglo XXI.

Oppenheim, P. and Putnam, H. 1958. The unity of science as a working hypothesis. In: H. Feigl et. al. (eds.) Minnesota Studies in the Philosophy of Science. Vol. 2. Minneapolis: Minnesota University Press.

Rosen, R. 1985. Organisms as causal systems which are not mechanisms: an essay into the nature of complexity. In: R. Rosen (ed.) Theoretical Biology and Complexity. Orlando: Academic Press.

Schrödinger, E. 1944. What is life? Cambridge: Cambridge University Press.

Shannon, C. E. 1948. A Mathematical theory of communication. Bell System Technical Journal 27: 379-423, and 623-56.

- 1949. Communication in the presence of noise. Proc. IRE 37: 10-21.

Szilard, L. 2003. On the decrease of entropy in a thermodynamic system by the intervention of intelligent beings. In: H. Leff; A. Rex (eds.) Maxwell's Demon: Entropy, Classical and Quantum Information, Computing. London: Institute of Physics Publishing.

Kourany, J. (ed.) 1987. Scientific Knowledge. Minneapolis: University of Minnesota Press.

Torres, J. M. 1993. Current Notions of Genotype and Phenotype Vis a Vis New Evidence in Molecular Biology. A Methodological Study. Biology Forum 86-2: 181-197.

Wicken, J.S . 1985. An Organism Critique of Molecular Darwinism. Journal of Theoretical Biology 117: 545-61.

- 1987. Entropy and Information: suggestions for common language. Philosophy of Science 54: 176-93.

Wiener, N. 1948. Cybernetics or Control and Communication in the Animal and the Machine. New York: John Wiley \& Sons.

- 1966. El hombre y la máquina. In: M. Gueroult (ed.) El concepto de información en la ciencia contemporánea. Coloquios de Royaumount. Barcelona: Siglo XXI.

Wiley, E. O.; Brooks, D.R. 1986. Evolution as entropy. Toward a unified theory of evolution. Chicago: Chicago University Press.

Zuckerland, E.; Pauling, L. 1965. Molecules as documents of evolutionary history. Journal of Theoretical Biology 8: 357-66.

\author{
JULIO ERNESTO RUBIO BARRIOS \\ Tecnológico de Monterrey \\ Calle del Puente 222 \\ Col. Ejidos de Huipulco \\ CP 14380 Tlalpan, México, D.F. \\ jerb@itesm.mx
}

\title{
Notes
}

${ }^{1}$ The argument is fundamentally the same one that Lwoff replicates before applying Shannon's equations to molecular genetics: the confusion between functional and structural order.

Principia 19(1): 121-146 (2015). 
${ }^{2}$ Social sciences, however, are not excluded from these structures of expectations, as can be shown when contemplating the cases of behaviorist programs in psychology, or the extensive use of statistical tools in sociological analysis.

${ }^{3}$ On the other hand, the nature of genetic information is a meta-theoretical problem which has been foreclosed in accordance to the specific practices in diverse fields of molecular biology, and it is for sure unknown to many scientists in the discipline. Molecular biology has become an established discipline which epistemic fundaments are robust. The localization of hereditary information in nucleic acids is an indisputable epistemological assumption, although evidence against this important dogma has built up (Torres, 1993). In spite of these emerging refutations, molecular biology is a well-placed scientific field regarding its theoretical knowledge (black boxes) and manipulation technics (technological objects). Not even the eventual collapse of its main dogma could appear as a threat to its stability, since it ceased to depend on the solidness of this criterion decades ago.

${ }^{4}$ These two problems are deeply connected, for to know the origin of life we must specify what live is.

${ }^{5}$ The computational characterization of biological systems seems to rest in the notion of the DNA as a generative program and in an analogy established with computer programs. This trend constitutes another epistemological source of technological inspiration to the concept of information.

Principia 19(1): 121-146 (2015). 